\title{
A systematic review and meta-analysis of the clinical efficacy of the intravenous injection of dexmedetomidine in ICU patients with hyperactive brain syndrome
}

\author{
Junna Wü, Bo Li", Kun Ma, Hong Li, Xian Shao \\ Department of Anesthesiology, the Fourth Hospital of Shijiazhuang, the Obstetrics and Gynecology Hospital of Hebei Medical University, \\ Shijiazhuang, China \\ Contributions: (I) Conception and design: J Wu, B Li, X Shao; (II) Administrative support: K Ma; (III) Provision of study materials or patients: J \\ Wu, B Li, K Ma, H Li; (IV) Collection and assembly of data: All authors; (V) Data analysis and interpretation: J Wu, B Li, X Shao; (VI) Manuscript \\ writing: All authors; (VII) Final approval of manuscript: All authors. \\ \#These authors contributed equally to this work. \\ Correspondence to: Xian Shao. Department of Anesthesiology, the Fourth Hospital of Shijiazhuang, the Obstetrics and Gynecology Hospital of Hebei \\ Medical University, No. 1 6, Tang Gu Bei Street, Chang'an District, Shijiazhuang, China. Email: liujiayi20080219@126.com.
}

\begin{abstract}
Background: The incidence of hyperactive brain syndrome in patients in the intensive care unit (ICU) is very high. Compared with other sedative drugs, existing research shows that dexmedetomidine can significantly reduce the incidence of hyperactive brain syndrome. This study systematically analyzed the clinical efficacy of a dexmedetomidine intravenous injection in ICU patients with hyperactive brain syndrome.

Methods: The databases PubMed, Web of Science, Embase, and the Cochrane Library were searched from January 2000 to December 2020 to identify papers that studied the clinical efficacy of dexmedetomidine intravenous injection in ICU patients with hyperactive brain syndrome. The basic information and evaluation indexes in the literature were screened and extracted. Revman5.3 software was used for quality assessment and meta-analysis of the included studies, and forest maps were drawn.
\end{abstract}

Results: A total of 255 patients were included in 5 studies. The results of the meta-analysis showed that intravenous infusion of dexmedetomidine could reduce the incidence of restless delirium in patients [odds ratio $(\mathrm{OR})=0.14 ; 95 \%$ confidence interval $(\mathrm{CI}): 0.07$ to 0.29 ; ( $\mathrm{Z}$ test) $\mathrm{Z}=5.39, \mathrm{P}<0.00001$ ], total delirium after medication duration [mean difference $(M D)=-15.50 ; 95 \%$ CI: -25.70 to $-5.29 ; Z=2.98 ; P=0.003$ ], and ICU hospitalization time ( $M D=-1.93 ; 95 \% \mathrm{CI}:-3.57$ to $-0.29 ; \mathrm{Z}=2.31 ; \mathrm{P}=0.02$ ). However, there was no significant difference in the incidence of adverse reactions (bradycardia and hypotension) in patients who were given an intravenous infusion of dexmedetomidine ( $\mathrm{OR}=2.85$; $95 \%$ CI: 0.21 to $38.74 ; \mathrm{Z}=0.79 ; \mathrm{P}=0.43$ ).

Discussion: The incidence of restlessness delirium, the duration of total delirium after medication, and the length of ICU stay in patients treated with a dexmedetomidine intravenous injection were significantly lower than those in patients treated with haloperidol, indicating that a dexmedetomidine intravenous injection had clinical efficacy in ICU patients with hyperactive brain syndrome.

Keywords: Dexmedetomidine; intravenous injection; ICU hyperactive brain syndrome; clinical efficacy; metaanalysis

Submitted Nov 29, 2021. Accepted for publication Jan 07, 2022.

doi: 10.21037/apm-21-3762

View this article at: https://dx.doi.org/10.21037/apm-21-3762 


\section{Introduction}

Hyperactive brain syndrome is a consciousness disorder characterized by acute onset and repeated fluctuation of attention loss, accompanied by changes in cognitive level or perceptual impairment that result in an impaired ability of patients to receive, process, store, and recall information $(1,2)$. The disease may be caused by factors such as neurophysiology and minor brain damage. Hyperactive brain syndrome is one of the most frequent complications in patients in the intensive care unit (ICU), with an incidence of between $4.9 \%$ and $91 \%$. The occurrence of hyperactive brain syndrome in patients in the ICU can cause many serious adverse outcomes. Its mortality and complication rates are significantly higher than those in patients with hyperactive brain syndrome, and the length of hospital stay, the length of stay in ICU, and the duration of mechanical ventilation were also significantly longer than those of hyperactive brain syndrome $(3,4)$. Hyperactive brain syndrome can also increase the risk of iatrogenic pneumonia by 9 times, it can greatly increase the incidence of complications such as aspiration, pulmonary infarction, and pressure ulcer, and it can increase the risk of accidental extubation and offline difficulty or re-endotracheal intubation after extubation in patients with mechanical ventilation (5).

The 2014 American Guide to Analgesia and Sedation in Critical Care Medicine pointed out the need for routine monitoring of hyperactive brain syndrome in adult ICU patients (6). Therefore, the monitoring and prevention of hyperactive brain syndrome has become an urgent and primary task for ICU doctors (7). At present, the sedative, analgesic, and hypnotic drugs most commonly used in the ICU not only achieve the ideal sedation and comfort of patients, but also frequently increase the number of patients with hyperactive brain syndrome and can increase the duration of hyperactive brain syndrome, therefore increasing the length of hospital stay and medical expenses for patients in the ICU. Many studies are still needed on the safety of these drugs (8). For patients with hyperactive brain syndrome, administering drugs in a timely manner has become the key to treating restless symptoms. However, reasonable selection of drug treatment in a timely manner has become a major challenge for ICU doctors (9). Therefore, drug treatment of hyperactive brain syndrome seems to be a double-edged sword.

Dexmedetomidine, as a new, powerful, and highly selective $\alpha_{2}$ receptor agonist, seems to have the above advantages.
Relevant studies have found that dexmedetomidine can significantly reduce the incidence of hyperactive brain syndrome compared with other sedative drugs, especially for patients with pulse poisoning (10). The 2014 American Guide to Analgesia and Sedation in Critical Care Medicine also recommends the use of dexmedetomidine. If the patient's delirium is not related to alcohol or benzodiazepine withdrawal, continuous intravenous infusion of dexmedetomidine rather than benzodiazepines is recommended for sedation to shorten the duration of delirium (11). In addition, recent studies have shown that dexmedetomidine can significantly shorten the time of mechanical ventilation in patients with hyperactive brain syndrome in the ICU compared with haloperidol (12). At present, research reports on the prevention of hyperactive brain syndrome are not rare, but there is still great controversy in the treatment of hyperactive brain syndrome, and there is no in-depth and accurate research to further clarify the comparison of the efficacy of dexmedetomidine (a new sedative) and haloperidol (a traditional antipsychotic) in the treatment of hyperactive brain syndrome in ICU patients (13).

At present, there are relatively few systematic evaluation studies on the treatment of dexmedetomidine in the treatment of ICU brain overactive syndrome. Based on this, our study collected relevant studies at home and abroad on the clinical efficacy of intravenous injection of dexmedetomidine in patients with hyperactive brain syndrome in the ICU and used quantitative methods to conduct a meta-analysis of the published literature. The clinical effect of intravenous injection of dexmedetomidine on patients with hyperactive brain syndrome in the ICU was determined to provide reference for the treatment of hyperactive brain syndrome in ICU patients. We present the following article in accordance with the PRISMA reporting checklist (available at https://apm.amegroups. com/article/view/10.21037/apm-21-3762/rc).

\section{Methods}

\section{Literature retrieval}

The literatures published in PubMed, Medline, Embase, CBM, and Wanfang Data from January 2000 to December 2020 were searched by computer. Literature was retrieved from the databases if it fulfilled the following criteria: (I) the subject and free words included a combination of "dexmedetomidine" and "hyperactive brain syndrome in 
ICU" the scope of search words was topic, keywords, and abstract; and (II) some references of the included literatures could be traced, and the full text could be manually retrieved and included in this study.

\section{Literature inclusion and exclusion criteria}

To be included in the meta-analysis, an article needed to fulfil the following inclusion criteria: (I) domestic and foreign published studies on the clinical efficacy of dexmedetomidine intravenous infusion in ICU patients with hyperactive brain syndrome; (II) clinical efficacy data could be obtained directly or indirectly; (III) at least 10 samples were included in the study; and (IV) was published in any language; literature research language was not limited.

Studies were excluded from the meta-analysis if they fulfilled the following exclusion criteria: (I) no original data was provided; (II) the study was a duplicate or was interdisciplinary literature; (III) the study included patients with bradycardia and ventricular block; (IV) the study included patients with hypotension; (V) the study included patients in a coma; and/or (VI) the study included patients with mental or nervous system diseases.

\section{Literature quality evaluation}

Two researchers used Revman5.3 (China) and a diagnostic test accuracy evaluation tool (QUADAS) to evaluate the quality of the included literature. When researchers disagreed, the third researcher conducted the intervention evaluation, and all three researchers finally reached an agreement through discussion. The evaluation criteria included case selection, methods to be evaluated, gold standard, evaluation process, and progress.

When the QUADAS tool was used to evaluate the included literature, it contained a total of 16 items and used "Yes", "No", and "Unclear" to evaluate the quality of the literature, where "Yes" meant conforming to the criteria and "No" meant not conforming to the criteria. A study was defined as "Unclear" when the information was not comprehensive or only partially met the criteria. Revman 5.3 was used to evaluate the literature quality, and the quality was determined from the following aspects: (I) whether it was a randomized controlled study; (II) whether there was allocation hiding; (III) whether a blind test was used; (IV) whether the resultant data was complete; (V) whether the study engaged in selective reporting; and (VI) whether there was any other bias.

\section{Data extraction}

Two researchers independently read the title and abstract of the retrieved literature and completed the extraction of the literature, obtained the full text of the included literature, and extracted the information. When researchers disagreed, they discussed their disagreement with each other. If no agreement was reached after discussion, the third researcher was required to determine the evaluation result.

The extracted data included the following: the name of the first author, the year of publication, research methods, the incidence of restless delirium, the total duration of delirium after medication, the length of stay in ICU, and adverse reactions (bradycardia, hypotension), etc. The above indicators were used to evaluate the therapeutic effect of dexmedetomidine. Researchers verified the data and then conducted systematic analysis.

\section{Statistical analysis}

Review Manager 5.3 (Cochrane Collaboration) software was used for data statistics and analysis. We evaluated only prospective studies that calculated the risk ratio of fracture occurrence and those studies that analyzed the data as a continuous variable and calculated a standard deviation difference. For studies that took place in the same location but with different participants, the risk ratio estimates were combined into a single estimate. The aggregation effect of the whole study was calculated. The main methods of sensitivity analysis included changing the inclusion criteria (especially for controversial studies), excluding low-quality studies, and using different statistical methods/models to analyze the same data. $\mathrm{I}^{2}$ described the percentage of changes in research caused by heterogeneity rather than contingency. $\mathrm{I}^{2}=0 \%$ indicated there was no heterogeneity. $\mathrm{I}^{2}=25 \%$ indicated mild heterogeneity. $\mathrm{I}^{2}=50 \%$ indicated moderate heterogeneity. $\mathrm{I}^{2}=75 \%$ indicated high heterogeneity. Due to heterogeneity, we used the random effect model in analysis. The funnel plot was drawn to analyze the publication bias of the included literature, and the symmetry of the funnel plot and the concentration of the sample to the midline were evaluated.

\section{Results}

\section{Literature search results and feature analysis}

A total of 878 records were retrieved from the database, and 856 abstracts related to this study were obtained after 


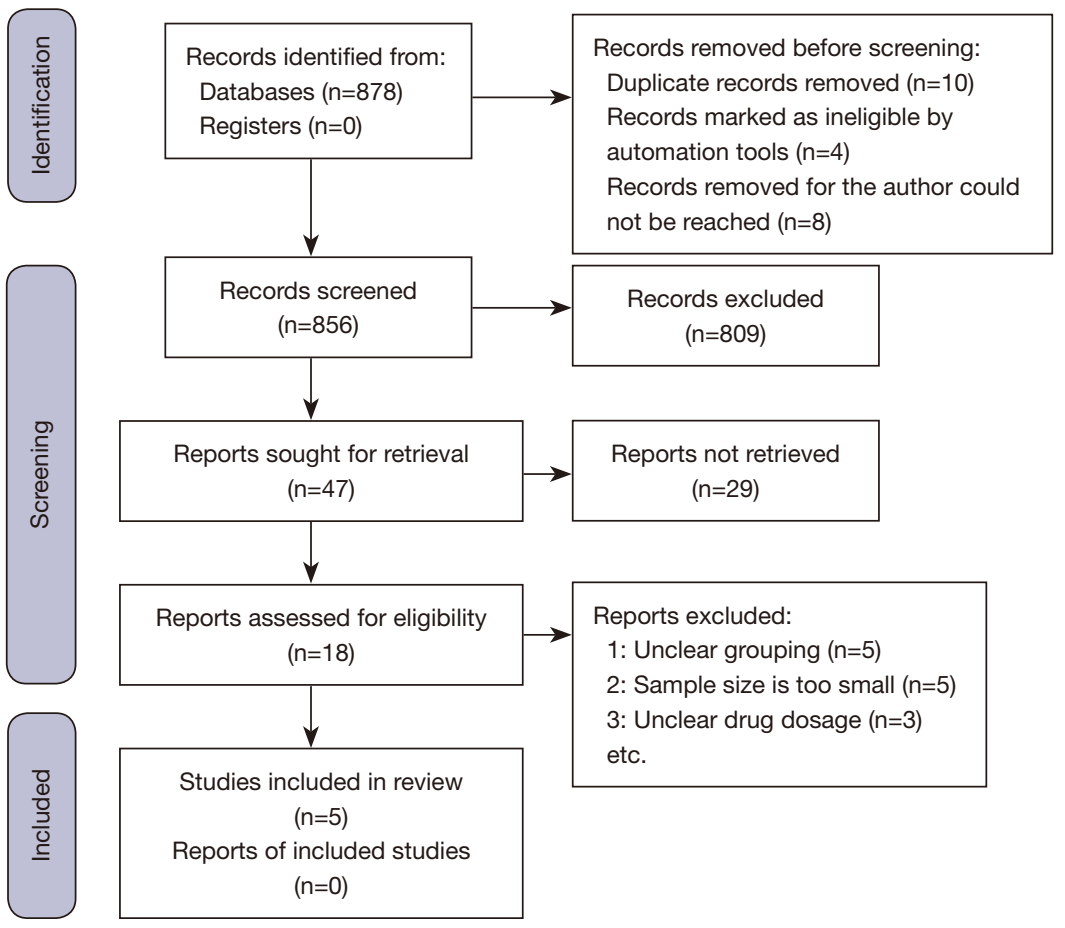

Figure 1 Flowchart showing the literature retrieval process.

Table 1 Basic features of literature included in this study

\begin{tabular}{|c|c|c|c|c|c|}
\hline First author & Year of publication & Research design & Total sample & \multicolumn{2}{|c|}{ Age (years old) } \\
\hline Essam (14) & 2016 & $\mathrm{RCT}$ & 60 & $26-70$ & $26-70$ \\
\hline Devlin (15) & 2014 & $\mathrm{RCT}$ & 33 & $66 \pm 6$ & $62 \pm 17$ \\
\hline Huang (16) & 2012 & $\mathrm{RCT}$ & 62 & $67.4 \pm 8.2$ & $61.5 \pm 7.3$ \\
\hline Anger (18) & 2010 & RCT & 56 & $63.71 \pm 11.48$ & $69 \pm 12.77$ \\
\hline
\end{tabular}

$\mathrm{RCT}$, randomized controlled trial.

deleting duplicate items. After reading the abstract and title of the article, two researchers preliminarily screened and obtained 47 literatures that met the requirements. After further reading of the full text of the literatures, nonrandom, repeated, and unavailable literatures were excluded, and five qualified literatures were included in this study (14-18). The literature retrieval process is shown in Figure 1, and the basic data of the literature included in the study are shown in Table 1.

\section{Quality evaluation of included literature}

Firstly, the bias risk assessment tool recommended by the Cochrane systematic review manual was used to evaluate the quality of the included literature (18). The results are shown in Figure 2 and Figure 3. The five included studies showed the largest percentage of "low risk" and "low concerns" overall, indicating that the included studies met the requirements of the analysis. 


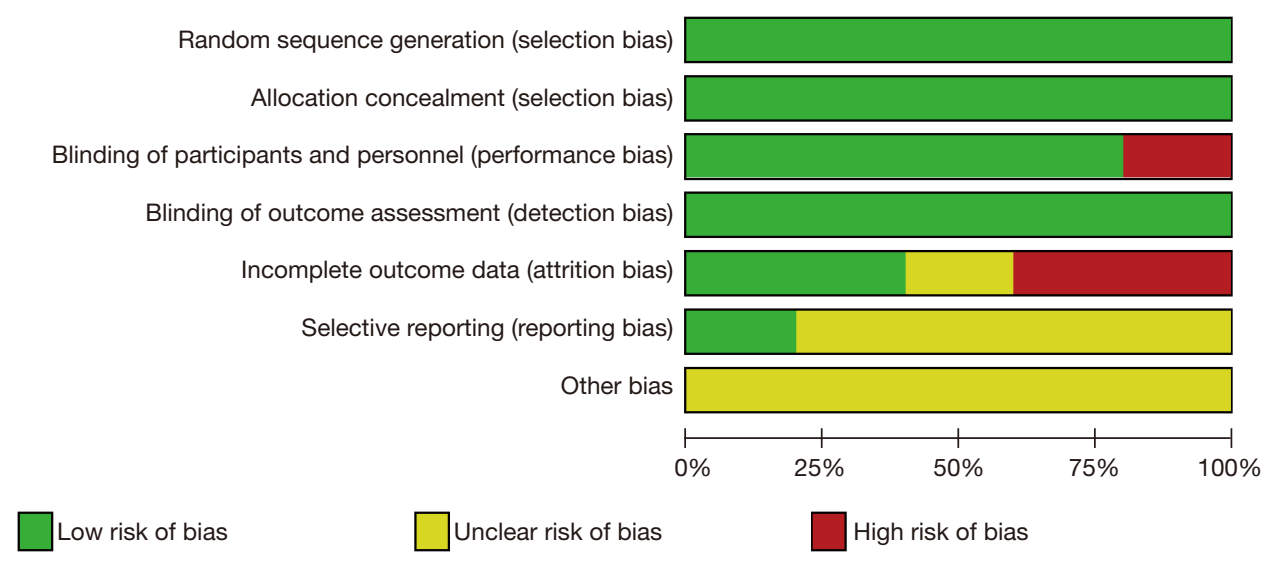

Figure 2 Bar chart showing the bias risk assessment for literature included in the study.

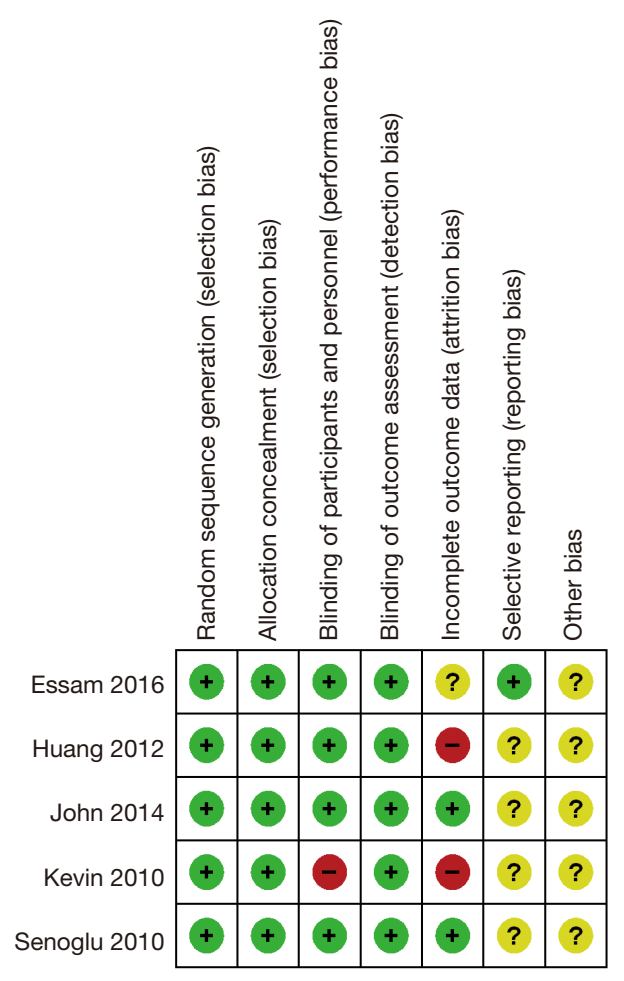

Figure 3 Summary of the bias risk assessment of literature included in the study. (Green: low risk, red: high risk, yellow: unclear).

QUADAS was used to evaluate the quality of each included literature. The results are shown in Table 2. The five literatures included in the study all have a low risk of bias, which meets the requirements of subsequent analysis.

\section{Results of meta-analysis}

\section{Incidence of restless delirium}

This study analyzed the relationship between the incidence of restless delirium in patients of the dexmedetomidine and haloperidol groups, as shown in Figure 4. Statistically, no significant heterogeneity was found between the incidence of restless delirium in the dexmedetomidine and haloperidol groups $\left(\mathrm{I}^{2}=0 \% ; \mathrm{P}=0.73\right)$, so the fixed-effect model was used for statistical analysis. The results showed that the effect value for the incidence of restless delirium in patients of the dexmedetomidine and haloperidol groups was OR $(95 \% \mathrm{CI})=0.14(0.07$ to 0.29$)$. The statistical value of the meta-analysis was $\mathrm{Z}=5.39$ and $\mathrm{P}<0.00001$. In summary, the mortality of patients in the dexmedetomidine group was significantly lower than that in the haloperidol group, and the difference was statistically significant $(\mathrm{P}<0.05)$.

\section{Total delirium duration after medication}

The relationship between the duration of total delirium in the dexmedetomidine group and haloperidol group was studied and analyzed. The results are shown in Figure 5. Results found that there was significant heterogeneity in the duration of total delirium between the dexmedetomidine group and the haloperidol group $\left(\mathrm{I}^{2}=98 \% ; \mathrm{P}<0.00001\right)$. Therefore, the random effect model was used for statistical analysis. The results showed that the effect value of the meta-analysis of total delirium duration in the dexmedetomidine group and the haloperidol group was MD $(95 \% \mathrm{CI})=-15.50(-25.70$ to -5.29$)$, and the statistical test structure was $\mathrm{Z}=2.98$ and $\mathrm{P}=0.003$. In summary, the 
Table 2 Bias risk assessment of literature included in the study

\begin{tabular}{|c|c|c|c|c|c|c|c|c|c|c|c|c|c|c|c|}
\hline First author & Year & 1 & 2 & 3 & 4 & 5 & 6 & 7 & 8 & 9 & 10 & 11 & 12 & 13 & 14 \\
\hline Devlin & 2014 & $\mathrm{Y}$ & $\mathrm{Y}$ & $Y$ & $U$ & $Y$ & $\mathrm{Y}$ & $\mathrm{Y}$ & $Y$ & Y & $Y$ & U & $Y$ & $\mathrm{Y}$ & $Y$ \\
\hline Huang & 2012 & $Y$ & $\mathrm{Y}$ & $Y$ & U & $Y$ & $Y$ & Y & $Y$ & Y & $\mathrm{Y}$ & $\mathrm{U}$ & $Y$ & $Y$ & $Y$ \\
\hline Senoglu & 2010 & Y & $Y$ & $Y$ & $U$ & $\mathrm{Y}$ & $\mathrm{Y}$ & Y & $Y$ & Y & U & $\mathrm{U}$ & $Y$ & $Y$ & $Y$ \\
\hline
\end{tabular}

1 to 14 are QUADAS item standards. $Y$, yes; U, unclear; QUADAS, quality assessment of diagnostic accuracy studies.

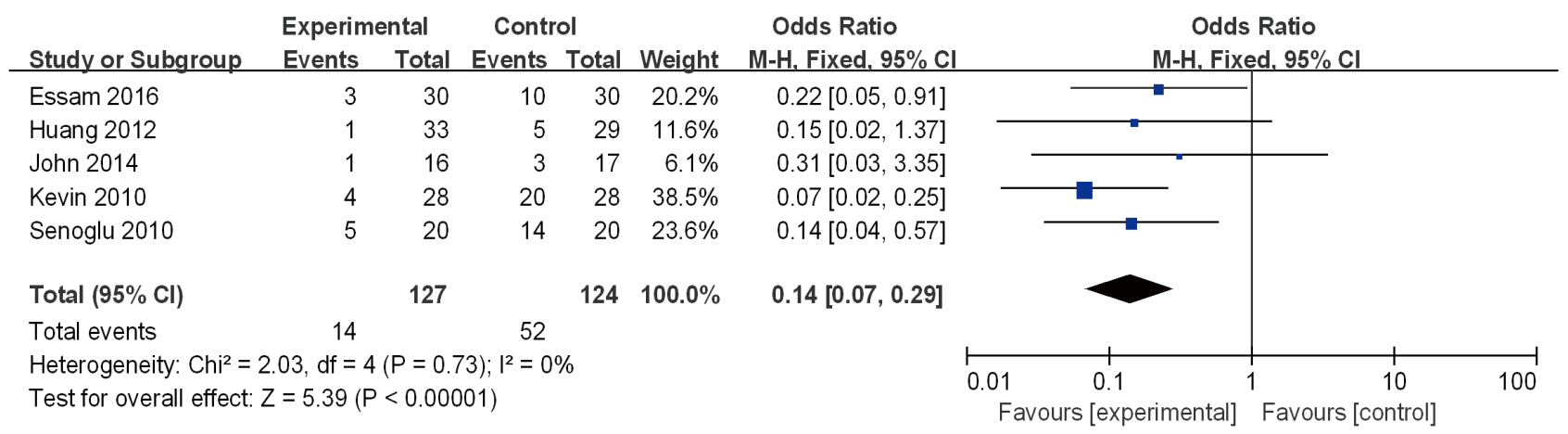

Figure 4 Forest map showing the comparison of restless delirium incidence. CI, confidence interval.

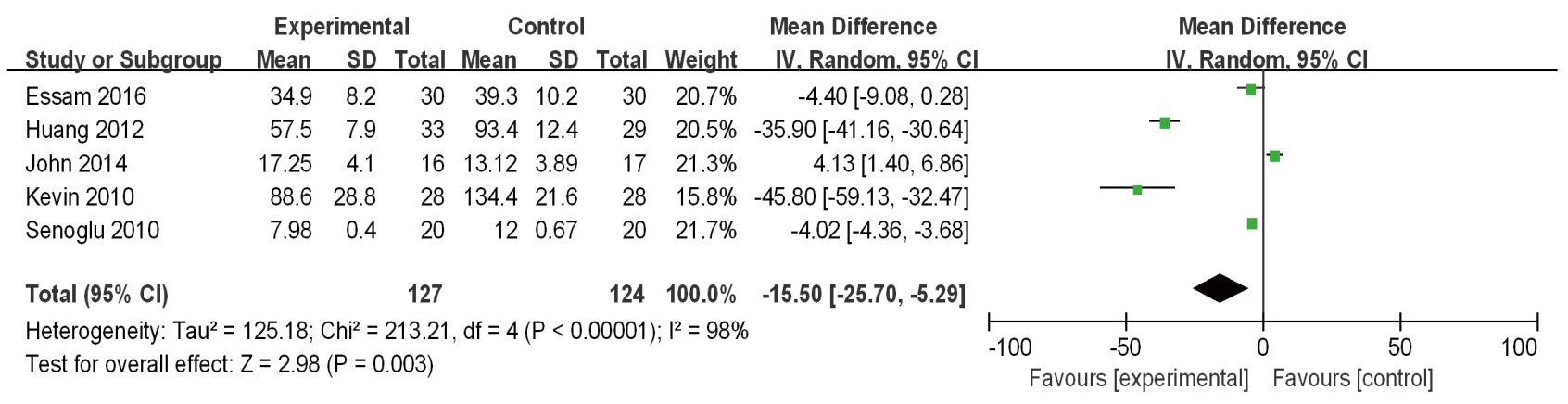

Figure 5 Forest map showing the comparison of total delirium duration after medication. CI, confidence interval; SD, standard deviation.

duration of total delirium in dexmedetomidine group was significantly lower than that in haloperidol group $(\mathrm{P}<0.05)$.

\section{ICU hospitalization time}

The relationship between ICU hospitalization time of patients in the dexmedetomidine group and the haloperidol group was studied and analyzed. The results are shown in Figure 6. Results found that there was significant heterogeneity in ICU hospitalization time between the dexmedetomidine group and the haloperidol group $\left(\mathrm{I}^{2}=91 \%\right.$; $\mathrm{P}<0.00001)$. Therefore, the random effect model was used for statistical analysis. The results showed that the effect value of the meta-analysis of ICU hospitalization time in the dexmedetomidine group and the haloperidol group was MD $(95 \% \mathrm{CI})=-1.93(-3.57$ to -0.29$)$, and the statistical test structure was $Z=2.31$ and $P=0.02$. The length of stay in ICU in the dexmedetomidine group was significantly lower than that in haloperidol group $(\mathrm{P}<0.05)$. 


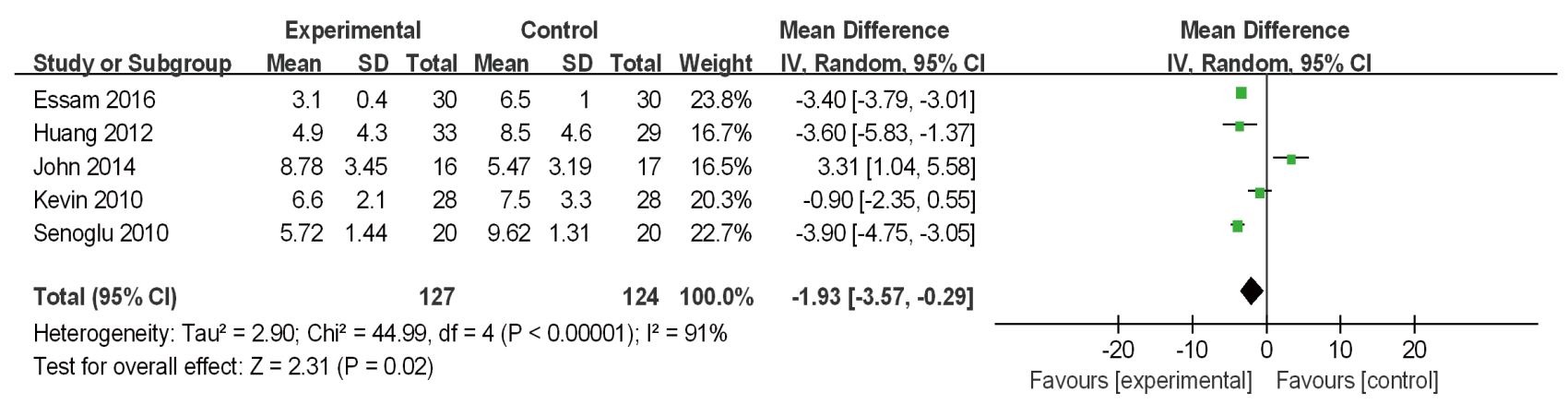

Figure 6 Forest map showing ICU hospitalization time comparison. CI, confidence interval; SD, standard deviation.

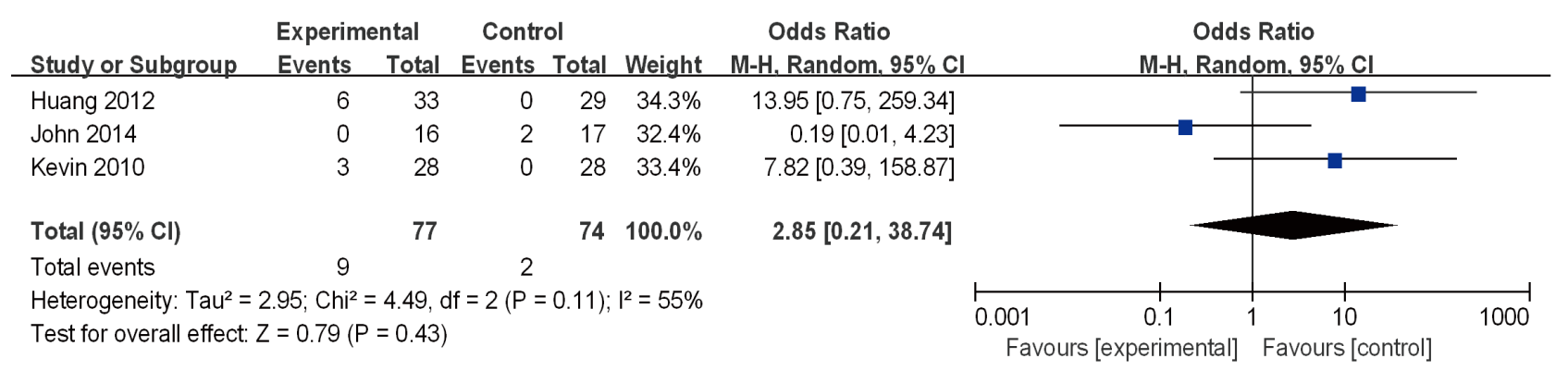

Figure 7 Forest map showing the comparison of adverse reactions (bradycardia; hypotension). CI, confidence interval.

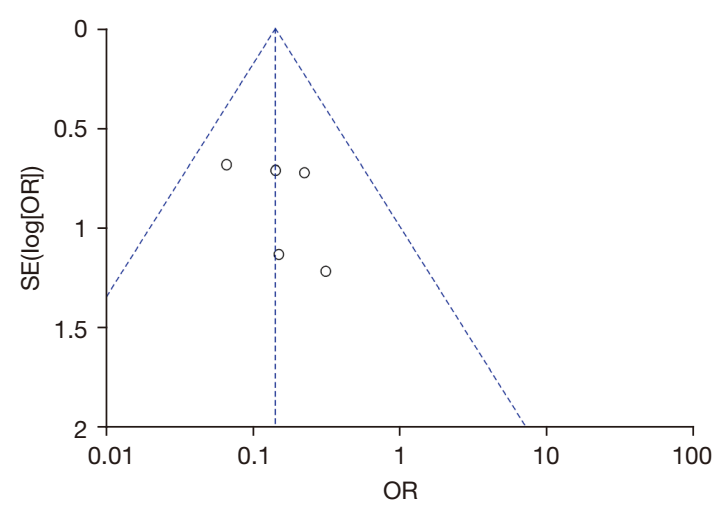

Figure 8 Funnel plot showing the incidence of restless delirium. SE, standard error; OR, odds ratio.

\section{Adverse reactions in ICU (bradycardia; hypotension)}

The relationship between adverse reactions (bradycardia and hypotension) in the dexmedetomidine group and the haloperidol group was analyzed. The results are shown in Figure 7. Results found that there was significant heterogeneity in the incidence of restless delirium between the dexmedetomidine group and the haloperidol group $\left(\mathrm{I}^{2}=55 \% ; \mathrm{P}=0.11\right)$. Therefore, the random effect model was used for statistical analysis. The results showed that the effect value of the meta-analysis of postoperative ICU residence time in the Dexmedetomidine group and haloperidol group was OR $(95 \% \mathrm{CI})=2.85$ ( 0.21 to 38.74$)$, and the statistical test structure was $\mathrm{Z}=0.79$ and $\mathrm{P}=0.43$. There was no significant difference in adverse reactions between the dexmedetomidine group and the haloperidol group $(\mathrm{P}>0.05)$.

\section{Publication bias analysis}

The statistically significant indexes of dexmedetomidine and haloperidol patients (the incidence of restless delirium), the total delirium duration after medication, and the publication bias of ICU hospitalization time were analyzed. The results are shown in Figures 8-10. The funnel diagram is relatively offset and not symmetrical. However, all the included documents fall into the figure and are close to the central axis. This shows that the publication bias of the literature included in this study is low and meets the requirements of the meta-analysis.

\section{Discussion}

In the ICU, severely ill patients often experience 


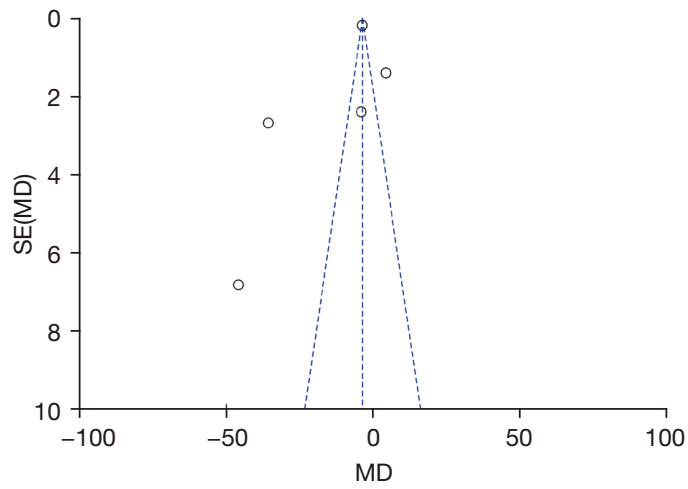

Figure 9 Funnel plot showing the total delirium duration after medication. SE, standard error; MD, mean difference.

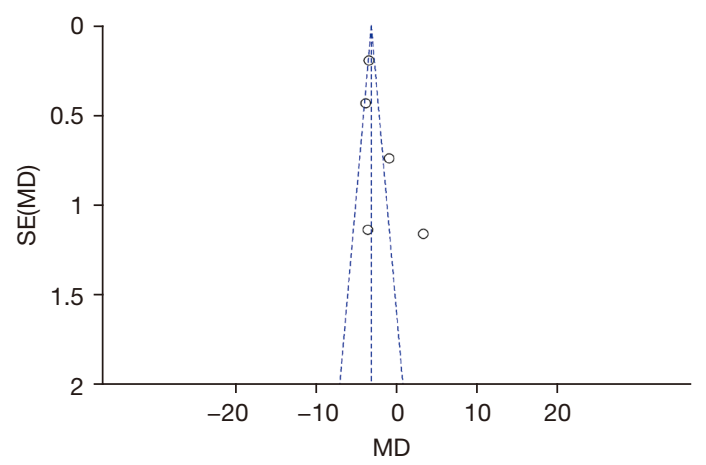

Figure 10 Funnel plot showing ICU hospitalization time. SE, standard error; MD, mean difference.

hyperactive brain syndrome due to many factors such as disease, environmental factors, and drug-related factors (19). The literature reports that the incidence of hyperactive brain syndrome in patients in the ICU is between $5.4 \%$ and $9.1 \%$ (20). Once patients have hyperactive brain syndrome in the ICU, intervention should take place and they should be treated as soon as possible to minimize the negative impact of subsequent complications (21). Comprehensive measures should be taken to treat hyperactive brain syndrome in patients in the ICU, including non-drug treatment and drug treatment (22). The incidence of hyperactive brain syndrome is high in patients in the ICU, and the occurrence of hyperactive brain syndrome is closely related to many serious adverse outcomes (23). Early detection and treatment of hyperactive brain syndrome is of great significance for ICU patients. Therefore, ICU medical staff should routinely monitor each patient with hyperactive brain syndrome $(24,25)$. Evaluation is conducted at least 3-4 times a day and details are recorded. This method is fast and simple and can enable ICU doctors to understand the consciousness and changes of patients in a short time. However, the specificity of the evaluation does not reach $100 \%$, and the use of this evaluation process is insufficient (26). Once hyperactive brain syndrome occurs in ICU patients, early intervention is needed. At present, the drug treatment for ICU patients with hyperactive brain syndrome still lacks evidential support $(27,28)$. Dexmedetomidine is a highly selective $\alpha 2$ adrenergic receptor agonist with fast onset, short action time, sedation, analgesia, and no respiratory depression. Recently, it has received more and more clinical attention (29). Dexmedetomidine achieves and maintains a sufficient level of sedation, while at the same time it has a unique feature of being easy to wake up, and at the same time, it can reduce the amount of other sedative drugs (30). The efficacy of antipsychotics such as haloperidol and dexmedetomidine in the treatment of hyperactive brain syndrome in ICU patients need to be further studied.

Our meta-analysis systematically analyzed the clinical efficacy of the dexmedetomidine intravenous injection in patients with hyperactive brain syndrome in the ICU. The results showed that the incidence of restless delirium, total delirium duration, and ICU hospitalization time of patients treated with an intravenous injection of dexmedetomidine were significantly lower than those treated with haloperidol, indicating that intravenous injection of dexmedetomidine has clinical effects on ICU patients with hyperactive brain syndrome.

Dexmedetomidine drugs can cause complications such as hypotension, heart rate slowing, and lung infections (31). Clinically, by adjusting the dosage of dexmedetomidine or stopping the administration, giving other vasodilators according to the situation, giving anticholinergic drugs, etc., can all improve the complications caused by drug treatment. The main disadvantage of our meta-analysis was that the sample sizes of included studies were small. More medium and large samples are needed to further subdivide the patients with different influencing factors into each subgroup to achieve a higher quality of results. If more factors are considered, the experimental results may be more accurate. Included studies also did not follow up with the patients who relapsed after the drug treatment, and the subjects were patients who were transferred out of the ICU without further follow-up observation. It is hoped that 
subsequent studies can improve on this limitation.

\section{Conclusions}

In order to systematically evaluate the clinical efficacy and safety of the dexmedetomidine intravenous injection in ICU patients with hyperactive brain syndrome, 5 related studies were included in a meta-analysis of the effects of dexmedetomidine and haloperidol on hyperactive brain syndrome in patients in the ICU. The results showed that an intravenous injection of dexmedetomidine could significantly reduce the incidence of restless delirium, the total duration of delirium, and the length of stay in ICU. However, this study only makes a systematic evaluation by using the existing studies. The results of this study can provide a foundation for the promotion of the intravenous injection of dexmedetomidine in the treatment of hyperactive brain syndrome in ICU patients.

\section{Acknowledgments}

Funding: None.

\section{Footnote}

Reporting Checklist: The authors have completed the PRISMA reporting checklist. Available at https://apm. amegroups.com/article/view/10.21037/apm-21-3762/rc

Conflicts of Interest: All authors have completed the ICMJE uniform disclosure form (available at https://apm. amegroups.com/article/view/10.21037/apm-21-3762/coif). The authors have no conflicts of interest to declare.

Ethical Statement: The authors are accountable for all aspects of the work in ensuring that questions related to the accuracy or integrity of any part of the work are appropriately investigated and resolved.

Open Access Statement: This is an Open Access article distributed in accordance with the Creative Commons Attribution-NonCommercial-NoDerivs 4.0 International License (CC BY-NC-ND 4.0), which permits the noncommercial replication and distribution of the article with the strict proviso that no changes or edits are made and the original work is properly cited (including links to both the formal publication through the relevant DOI and the license). See: https://creativecommons.org/licenses/by-nc-nd/4.0/.

\section{References}

1. Romagnoli S, Amigoni A, Blangetti I, et al. Light sedation with dexmedetomidine: a practical approach for the intensivist in different ICU patients. Minerva Anestesiol 2018;84:731-46.

2. Atterton B, Paulino MC, Povoa P, et al. Sepsis Associated Delirium. Medicina (Kaunas) 2020;56:240.

3. Cortés-Beringola A, Vicent L, Martín-Asenjo R, et al. Diagnosis, prevention, and management of delirium in the intensive cardiac care unit. Am Heart J 2021;232:164-76.

4. Zaal IJ, Devlin JW, Peelen LM, et al. A systematic review of risk factors for delirium in the ICU. Crit Care Med 2015;43:40-7.

5. Ganau M, Lavinio A, Prisco L. Delirium and agitation in traumatic brain injury patients: an update on pathological hypotheses and treatment options. Minerva Anestesiol 2018;84:632-40.

6. Humble SS, Wilson LD, Leath TC, et al. ICU sedation with dexmedetomidine after severe traumatic brain injury. Brain Inj 2016;30:1266-70.

7. He X, Cheng KM, Zhang L, et al. Dexmedetomidine for the prevention of postoperative delirium in patients after intracranial operation for brain tumours (DEPOD study): a study protocol and statistical plan for a multicentre randomised controlled trial. BMJ Open 2020;10:e40939.

8. Shelton KT, Qu J, Bilotta F, et al. Minimizing ICU Neurological Dysfunction with Dexmedetomidineinduced Sleep (MINDDS): protocol for a randomised, double-blind, parallel-arm, placebo-controlled trial. BMJ Open 2018;8:e20316.

9. Choi HA, Ko SB, Presciutti M, et al. Prevention of shivering during therapeutic temperature modulation: the Columbia anti-shivering protocol. Neurocrit Care 2011;14:389-94.

10. Yang G, Guo X, Mao G, et al. Clinical research of early goal directed sedation applying in acute brain injury. Zhonghua Wei Zhong Bing Ji Jiu Yi Xue 2020;32:345-9.

11. Piva S, McCreadie VA, Latronico N. Neuroinflammation in sepsis: sepsis associated delirium. Cardiovasc Hematol Disord Drug Targets 2015;15:10-8.

12. Huang W, Qin Y, Dai X. Breakthroughs in global critical care medicine 2019. Zhonghua Wei Zhong Bing Ji Jiu Yi Xue 2020;32:1-7.

13. Horner D, Altshuler D, Droege C, et al. Major publications in the critical care pharmacotherapy literature: January-December 2016. J Crit Care 2018;43:327-39.

14. Essam FA. Dexmedetomidine versus haloperidol for 
prevention of delirium during non-invasive mechanical ventilation. Egypt J Anaesth 2016;32:473-81.

15. Devlin JW, Al-Qadheeb NS, Chi A, et al. Efficacy and safety of early dexmedetomidine during noninvasive ventilation for patients with acute respiratory failure: a randomized, double-blind, placebo-controlled pilot study. Chest 2014;145:1204-12.

16. Huang Z, Chen YS, Yang ZL, et al. Dexmedetomidine versus midazolam for the sedation of patients with noninvasive ventilation failure. Intern Med 2012;51:2299-305.

17. Senoglu N, Oksuz H, Dogan Z, et al. Sedation during noninvasive mechanical ventilation with dexmedetomidine or midazolam: A randomized, double-blind, prospective study. Curr Ther Res Clin Exp 2010;71:141-53.

18. Anger KE, Szumita PM, Baroletti SA, et al. Evaluation of dexmedetomidine versus propofol-based sedation therapy in mechanically ventilated cardiac surgery patients at a tertiary academic medical center. Crit Pathw Cardiol 2010;9:221-6.

19. Hipp DM, Ely EW. Pharmacological and nonpharmacological management of delirium in critically ill patients. Neurotherapeutics 2012;9:158-75.

20. Tang JF, Chen PL, Tang EJ, et al. Dexmedetomidine controls agitation and facilitates reliable, serial neurological examinations in a non-intubated patient with traumatic brain injury. Neurocrit Care 2011;15:175-81.

21. Wenjie W, Houqing L, Gengyun S. Effects of dexmedetomidine on outcomes following craniocerebral operation - a meta-analysis. Clin Neurol Neurosurg 2014;125:194-7.

22. Bienvenu OJ, Neufeld KJ, Needham DM. Treatment of four psychiatric emergencies in the intensive care unit. Crit Care Med 2012;40:2662-70.

23. Erkonen G, Lamb F, Tobias JD. High-dose dexmedetomidine-induced hypertension in a child with traumatic brain injury. Neurocrit Care 2008;9:366-9.

Cite this article as: $\mathrm{Wu} \mathrm{J}, \mathrm{Li} \mathrm{B}, \mathrm{Ma} \mathrm{K}, \mathrm{Li} \mathrm{H}$, Shao X. A systematic review and meta-analysis of the clinical efficacy of the intravenous injection of dexmedetomidine in ICU patients with hyperactive brain syndrome. Ann Palliat Med 2022;11(1):299308. doi: 10.21037/apm-21-3762
24. Cascella M, Fiore M, Leone S, et al. Current controversies and future perspectives on treatment of intensive care unit delirium in adults. World J Crit Care Med 2019;8:18-27.

25. Aryan HE, Box KW, Ibrahim D, et al. Safety and efficacy of dexmedetomidine in neurosurgical patients. Brain Inj 2006;20:791-8.

26. Tsaousi GG, Lamperti M, Bilotta F. Role of Dexmedetomidine for Sedation in Neurocritical Care Patients: A Qualitative Systematic Review and Metaanalysis of Current Evidence. Clin Neuropharmacol 2016;39:144-51.

27. Rajan R, Sage M. Successful Emergency Treatment of Refractory Neuroleptic Malignant Syndrome With Electroconvulsive Therapy and a Novel Use of Dexmedetomidine: A Case Report From California in the Era of COVID-19. J ECT 2021;37:71-3.

28. Fiore M, Torretta G, Passavanti MB, et al. Dexmedetomidine as adjunctive therapy for the treatment of alcohol withdrawal syndrome: a systematic review protocol. JBI Database System Rev Implement Rep 2019;17:2159-64.

29. Barends CR, Absalom A, van Minnen B, et al. Dexmedetomidine versus Midazolam in Procedural Sedation. A Systematic Review of Efficacy and Safety. PLoS One 2017;12:e0169525.

30. Kawazoe Y, Miyamoto K, Morimoto T, et al. Effect of Dexmedetomidine on Mortality and Ventilator-Free Days in Patients Requiring Mechanical Ventilation With Sepsis: A Randomized Clinical Trial. JAMA 2017;317:1321-8.

31. Mahmoud M, Mason KP. Dexmedetomidine: review, update, and future considerations of paediatric perioperative and periprocedural applications and limitations. Br J Anaesth 2015;115:171-82.

(English Language Editor: C. Mullens) 